\title{
Failure to Find Altruistic Food Sharing in Rats
}

\author{
Haoran Wan ${ }^{1}$, Cyrus Kirkman', Greg Jensen ${ }^{2,3}$, and Timothy D. Hackenberg ${ }^{1,4}$ \\ ${ }^{1}$ Dept. of Psychology, Reed College \\ ${ }^{2}$ Dept. of Neuroscience, Columbia University \\ ${ }^{3}$ Zuckerman Mind Brain Behavior Institute, Columbia University \\ ${ }^{4}$ Corresponding author (hack@reed.edu)
}

\section{ABSTRACT}

Prior research has found that one rat will release a second rat from restraint in the presence of food, thereby allowing that second rat access to food. Such behavior, clearly beneficial to the second rat and costly to the first, has been interpreted as altruistic. Because clear demonstrations of altruism in rats are rare, such findings deserve a careful look. The present study aimed to replicate this finding, but with more systematic methods to examine whether, and under what conditions, a rat might share food with its cagemate partner. Rats were given repeated choices between high-valued food (sucrose pellets) and 30-s social access to a familiar rat, with the (a) food size (number of food pellets per response), and (b) food motivation (extra-session access to food) varied across conditions. Rats responded consistently for both food and social interaction, but at different levels and with different sensitivity to the food-access manipulations. Food production and consumption was high when food motivation was also high (food restriction) but substantially lower when food motivation was low (unlimited food access). Social release occurred at moderate levels, unaffected by the food-based manipulations. When food was abundant and food motivation low, the rats chose food and social options about equally often, but sharing (food left unconsumed prior to social release) occurred at low levels across sessions and conditions. Even under conditions of low food motivation, sharing occurred on only $1 \%$ of the sharing opportunities. The results are therefore inconsistent with claims in the literature that rats are altruistically motivated to share food with other rats.

Keywords: social reward, food reward, preference, altriusm, lever press, rats

Pro-social behavior has been defined as behavior that produces benefits for another, sometimes even at a cost to the individual (Cronin, 2012; Sosnowski and Brosnan, 2019; West et al., 2007). One type of pro-social behavior gaining currency in recent years is social release, in which one animal releases another from a trap or restraint. In an experiment by Ben-Ami Bartal et al. (2011), for example, one rat was restrained in a plastic restraint tube that could be opened by a second rat. Such release permitted the restrained animal to leave the tube and spend the remainder of the 60-min session in the presence of the other rat. Most of the rats (17 of 23) learned to open the restraint after an average of about seven sessions. Subsequent studies have verified that rats will, under a variety of conditions, respond in ways that release a rat from a restraint (Ben-Ami Bartal et al., 2014; Blystad et al., 2019; Hachiga et al., 2018; Hiura et al., 2018; Sato et al., 2015; Schwartz et al., 2017; Silberberg et al., 2014; Vanderhooft et al., 2019). The basic effect is reliable, having been replicated across different procedures and laboratories, but its core mechanisms remain a matter of debate.

According to some authors (Ben-Ami Bartal et al., 2011, 2014; Sato et al., 2015), social release arises from altruistic motives: the free rat senses distress on the part of the trapped rat, and acts altruistically out of empathic concern for its social partner. An alternative explanation is based on social contact: social release is motivated by opportunities for social interaction (Hachiga et al., 2018; Hiura et al., 2018; 
Schwartz et al., 2017; Vanderhooft et al., 2019). In other words, social release is a type of operant (or instrumental) behavior, established and maintained by contingent social contact as a form of reinforcement. The competing theoretical accounts have been difficult to disentangle experimentally, owing to the fact that under many conditions in the standard procedure, releasing the other rat from a restraint can be viewed in terms consistent with either a social reinforcement view (opportunities for social interaction) or an altruistic view (releasing a distressed rat for its benefit, rather than that of the releaser). Therefore, unless special conditions are arranged to disentangle the two interpretations (cf. Hachiga et al., 2018; Sato et al., 2015; Schwartz et al., 2017), the mere fact of social release is not sufficient to favor one view over the other.

Although most of the work to date on social-release procedures has focused on the main procedure, another result reported by Ben-Ami Bartal et al. (2011) has received far less empirical attention. In some conditions, rats were given a choice between two restrainers, one of which contained high-value food rewards ( 5 chocolate chips), the other of which was contained a restrained rat. These conditions permitted social release, as in the standard procedure, but here, was pitted against a known and powerful food reward as a means of assessing the relative value of social release. On average, the rats learned to approach and open the tube containing food more quickly (earlier in testing) than the tube containing another rat, though the latencies to access both tubes became low and roughly comparable $(<10 \mathrm{~s})$ by the end of the 12-session experiment. And on slightly more than half of the trials, the trapped rat was released before the food was completely consumed, enabling the trapped rat access to the food rewards. This resulted in lower levels of food intake ( $70 \%$ of maximum, on average) than in a control condition in which the alternative restraint was empty (in which the free rats ate $96 \%$ of the food).

These two patterns of findings led the authors to the following conclusions: (a) the reward value of releasing a restrained rat is comparable to that of high-valued food (similar latencies to food and social release doors), and (b) social release in some cases comes at the expense of food intake (lower levels of food intake in a social context). In other words, rats not only value social contact equally with food, but engage in altruistic food sharing, sacrificing some high-value food to make it available to a distressed social partner. On its face, these findings appear to lend strong support to accounts appealing to some type of altruism, and simultaneously pose serious theoretical challenges to strict cost-benefit models. If not for altruistic motives, why would a rat sacrifice available food for the good of another? While there is ample evidence of food sharing in rats, it is usually of the reciprocal exchange (tit-for-tat) variety, in which animals alternate roles as donors and receivers (Taborsky et al., 2016). Far less common is the type of unreciprocated food sharing reported by Ben-Ami Bartal et al. (2011), in which a rat leaves highly-valued food for another to consume with no tangible short-term gain for itself. Given both the novelty and theoretical significance of the findings, these food-sharing conditions warrant closer examination.

The main objective of the present study was to replicate and extend the food-sharing conditions from Ben-Ami Bartal et al. (2011), using more robust methods for assessing reward value and food sharing. The methods were patterned after Hiura et al. (2018), in which rats were given repeated choices between high-valued food rewards (sucrose pellets) and social release (10-s social contact). Unlike the findings reported by Ben-Ami Bartal et al., however, Hiura et al. found that rats showed a consistently strong preference for food over social release, even in the face of large increases in food cost (number of responses to produce food), while the costs of social release remained low. Costs were manipulated via a progressive ratio (PR) schedule, in which the number of responses per reward was low at the beginning of the session, but increased with each reward earned. Social release typically only occurred in the latter parts of the session, when food costs were high and after many food rewards had been earned and consumed. The overall session-wide preference for food generally exceeded $90 \%$.

This strong preference for the food over the social release option is at odds with the equal value 
of social release and food reported by Ben-Ami Bartal et al. (2011). There are several procedural differences between the experiments, however, that may account for the different findings. First, and perhaps importantly, there were differences in motivation. In the Ben-Ami Bartal et al. experiment, there were no restrictions placed on social or food access outside the session: rats had free access to food and social contact in their homecages. In the Hiura et al. (2018) experiment, on the other hand, food or social access (or both) were generally restricted outside the session. When food was freely available outside the session, the rats still preferred food over social contact, but substantially less so than when it was restricted, suggesting some sensitivity to the motivational context. In the present experiment, we included conditions that both restricted and did not restrict access to food outside the sessions to assess the impact of motivational variables on the relative value of food and social rewards. If the higher levels of food versus social responding reported by Hiura et al. (2018) are due primarily to motivational variables (restricted post-session access to rewards), then providing free access to those rewards would be expected, through satiation, to reduce the reward value of food, bringing food preferences more in line with social preference, akin to findings of Ben-Ami Bartal et al. (2011).

We also sought a more detailed characterization of altrusitic food sharing, the second important claim put forth by Ben-Ami Bartal et al. (2011). In their study, sharing was defined in terms of differences in food consumed when a rat was available for release and when no rat was available (empty tube), differences thought to reflect the amount of food that is consumed (hence, shared) with the other rat. While this provides a tangible measure of some important outcomes of sharing, it has little to say about the functional characteristics of the sharing behavior itself, or the conditions under which it occurs. In the present experiment, we adopted a functional definition of altruistic food sharing, focusing on a coordinated sequence of behavior: (a) food production, followed by (b) social release, given that (c) food was still remaining, permitting (d) the formerly restrained rat access to food. Measuring such episodes of food sharing alongside preference for food and social release will provide important information on how these behavior patterns are related to each other as well as to the experimental conditions.

In addition, to ensure that the choices were well informed by their outcomes, we gave rats repeated choices each session, rather than the single choice per session used by Ben-Ami Bartal et al. (2011). In single-choice procedures, the duration of social contact depends on when in the session the door opening occurs. If the duration of social contact contributes to reward value, as some research suggests (Vanderhooft et al., 2019), then the value of social release in these single-choice procedures may fluctuate across sessions; and this may, in turn, affect sharing opportunities. We sought to hold constant the value of social contact, both within and across sessions, and therefore used a consistent (30 s) duration of social contact throughout the present experiment. Against this background of constant value of social reward, we manipulated food quantity (pellets per response) and motivational context (restricted versus unrestricted food access) on a within-subject basis, including some conditions closely resembling the original procedure used by Ben-Ami Bartal et al. Collectively, the methods permit a rigorous evaluation of Ben-Ami Bartal's two main conclusions bearing on their claim of altruistic food sharing, namely, that (1) reward value of food and social release are equal, and (2) a rat will share food with another rat, even at the expense of food for itself.

\section{METHODS}

\section{Subjects}

Six female Sprague-Dawley rats (Rattus norvegicus) were used in this experiment. The rats were experimentally naïve, and were pair-housed in in Ancare ${ }^{\circledR}$ transparent polycarbonate rodent cages (measuring $26.5 \mathrm{~cm} \times 48.2 \mathrm{~cm} \times 20.3 \mathrm{~cm}$ ) in a temperature-controlled colony room, with a 12-hr 


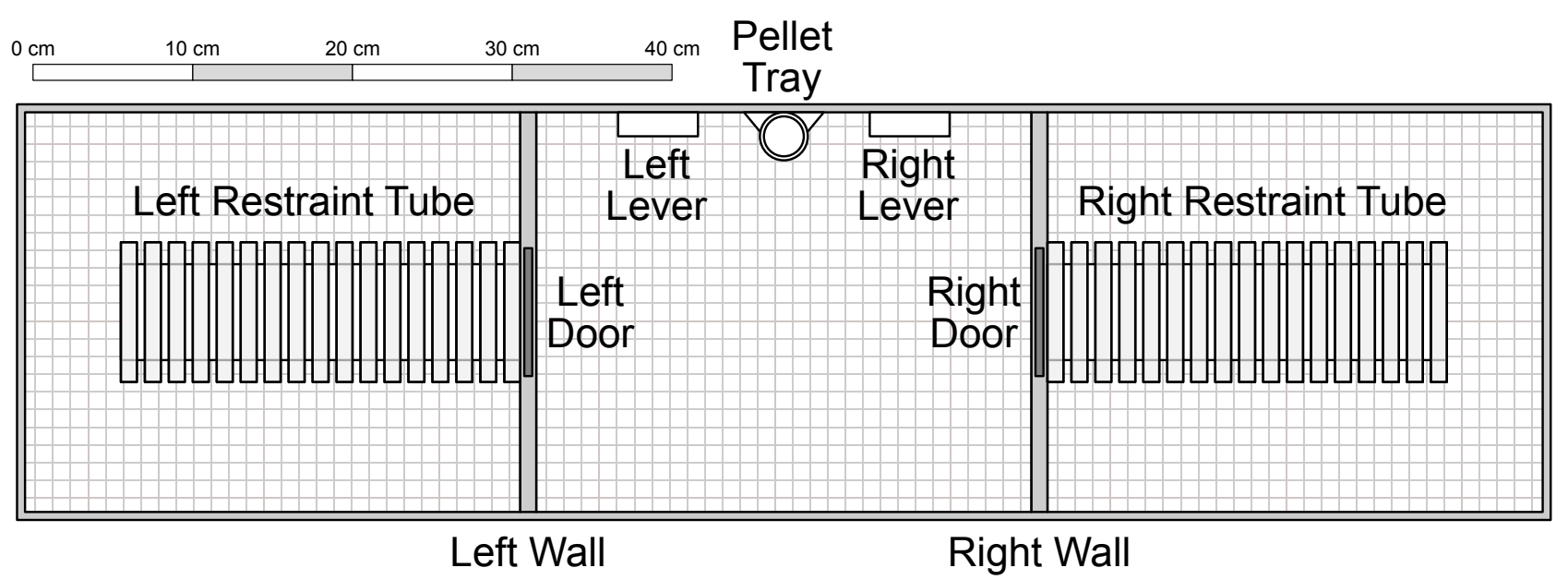

Figure 1. Experimental apparatus, with components presented to scale. The inner left and right walls consisted of opaque plastic. The restraint tubes were cylinders composed of clear Plexiglas. Experimenters accessed each of the three chambers through clear Plexiglas ceiling hatches that acted as a roof when closed. Not shown are two indicator lights set into the wall immediately above each of the levers. Unless otherwise noted, components were composed of aluminum. In Conditions 1-4, pellets were delivered into the pellet tray from a dispenser outside the apparatus. In Conditions 5-7, food was dispensed into the right restraint tube.

light/dark cycle. One rat from each pair designated the unrestrained (focal) rat and the other the restrained rat. The rats had continuous homecage access to water whereas the availability of food (Purina ${ }^{\circledR}$ Rat Diet 5012) was sometimes restricted (see below).

\section{Apparatus}

The apparatus consisted of three adjacent chambers, each measuring $31 \mathrm{~cm} \times 25 \mathrm{~cm} \times 22 \mathrm{~cm}$ with a wire grid floor. The central chamber contained two levers $(5 \mathrm{~cm} \times 1.5 \mathrm{~cm} \times 1.5 \mathrm{~cm})$ set on either side of a pellet tray, which could receive individual pellets from a gravity-fed dispenser outside the chamber. Additionally, a small light ( $2 \mathrm{~cm}$ diameter) was mounted above each lever. Both the left and right chambers contained a Plexiglas rodent restrainer $\left(25 \mathrm{~cm} \times 8.75 \mathrm{~cm} \times 7.5 \mathrm{~cm}\right.$, Harvard Apparatus $\left.{ }^{\circledR}\right)$, each of which was connected to the central chamber by a metal door that was mechanically controlled. Experimenters were able to access each chamber separately via a hatch that, when closed, acted as a ceiling to the chamber, Experimental schedules were controlled and data recorded via a PC computer programmed in the MedState Notation language and MED-PC ${ }^{\circledR}$ software.

\section{Training}

\section{Food reinforcement training}

Focal rats were trained to press the right lever by reinforcing successive approximations with food, delivered into the pellet tray (see Figure 1), accompanied by a $0.5 \mathrm{~s}$ auditory tone. Only the right lever was active in these sessions, denoted by the illumination of the right cue light.

\section{Restraint training}

To minimize the delay between lever pressing and social interaction, restrained rats were trained to leave the restraint soon after the door was opened. During these sessions, the restraint door was lifted independently of a subject's behavior. Once the rat left the restraint (defined by its entire body, except the 
tail, having passed through the door) and entered the middle chamber, it was allowed to explore for $30 \mathrm{~s}$ before being returned to the restrainer by hand for the next trial. These sessions lasted for $30 \mathrm{~min}$, and continued for 3-5 sessions, until the rats were leaving consistently within a few seconds of when the door was raised.

\section{Social reinforcement training}

After food and restraint training was complete, all rats received social reinforcement training. In these sessions, the restrained rat began each trial in the restraint in the left chamber, with the focal rat in the center chamber. Only the left lever was active in these sessions, denoted by the left cue light. A left lever press opened the door to the restraint, and produced a 2.0-s tone. When the restrained rat moved from the restraint into the center chamber, the door was closed, beginning the 30-s social interaction period. This also extinguished the light and deactivated the lever. After $30 \mathrm{~s}$, the restrained rat was returned to the restraint for the next trial.

Rat 4 pressed the social lever in the first training session, and therefore required no additional training; left lever presses only produced social access throughout the experiment. Rats 6 and 8 did not readily press the left lever for social access, and so received two sessions of supplemental training in which left lever presses produced food. For Rat 6, this brief training was sufficient to establish social responding, and the food contingency on the left lever was withdrawn thereafter. For Rat 8, the food training was also effective in establishing left lever pressing, but responses for social access alone were minimal, compared to the right-lever food responses. To induce more left-lever responses, the contingencies for right-lever responses were made less favorable: 100 responses per food reward (6 sessions) and no rewards/extinction (4 sessions). These changes were successful in producing more frequent social responding, and thereafter the additional contingencies for right-lever responding were discontinued, and the experiment proper began.

\section{Experimental Procedure}

During all experimental sessions, the rat in the central chamber made repeated concurrent choices between social release and food, unless otherwise noted. With the exception of Condition 5, choice trials began with both left and right cue lights illuminated and a restrained rat occupied the left restraint. A press on the right (food) lever produced food, with the food quantity, food motivation, and food location varied across conditions (see below). A press on the left (social release) lever opened the left door for a 30-s social interaction period, while also deactivating both levers (and their associated cue lights) for the duration of the interaction period. This prevented the restrained rat from producing consequences arranged for the focal rat. At the end of the social interaction period, the apparatus was reset for the next trial, with the restrained rat returned to the left restraint while the focal rat remained in the central chamber. Sessions lasted for a total of 30 min and were conducted five days per week.

A within-subject experimental design was employed, wherein each subject was exposed to experimental conditions in which the main independent variables (food quantity, motivation, food location) were systematically manipulated across blocks of sessions. Table 1 shows the combination of parameters constituting each of the 7 experimental conditions and the sequence in which they were arranged. In Conditions 1-4, food quantity (pellets per press) was increased systematically across conditions: one

pellet in Condition 1, two pellets in Condition 2, and four pellets in Conditions 3 and 4. The quantity of food was held constant at 5 pellets per press in Conditions 5-7. Homecage access to food was varied across conditions by restricting access to food to a period of $60 \mathrm{~min}$ following the session (Conditions 1-3), or permitting unlimited homecage access (Conditions 4-7).

The location and accessibility of food varied across conditions. In Conditions 1-4, food was dispensed 


\begin{tabular}{c|c|c|c|c|c|c} 
Condition & $\begin{array}{c}\text { Pellets per response } \\
\text { (location of food) }\end{array}$ & $\begin{array}{c}\text { Home Cage } \\
\text { Chow }\end{array}$ & $\begin{array}{c}\text { Food Collection } \\
\text { Period }\end{array}$ & \multicolumn{3}{|c}{ \# of Sessions } \\
\hline 1 & 1 R4 & R6 & R8 \\
\hline 2 & 2 (Tray) & Restricted & N/A & 11 & 11 & 5 \\
3 & 4 (Tray) & Restricted & N/A & 9 & 9 & 4 \\
4 & 4 (Tray) & Unrestricted & N/A & 13 & 9 & 5 \\
5 & 5 (Right Tube) & Unrestricted & 30 s & 7 & 5 & 8 \\
6 & 5 (Right Tube) & Unrestricted & Unlimited & 6 & 6 & 6 \\
7 & 5 (Right Tube) & Unrestricted & $30 s$ & 6 & 7 & 5
\end{tabular}

Table 1. Sequence of conditions and number of sessions conducted at each.

in the pellet tray, in close proximity to the lever, whereas in Conditions 5-7, the food was made available in the restraint tube behind the right door. In these latter conditions, a right lever press opened the right door for a food collection period. In Conditions 5 and 7, this food collection period was $30 \mathrm{~s}$, after which the door closed whether or not all pellets had been consumed. In Condition 6, however, the door remained open until all pellets had been consumed (by either rat). In Condition 5, only the food lever was active; there was no rat in the left restraint, the left cue light was left dark, and left lever presses had no mechanical effects.

The main dependent variables were (1) proportions of food and social choices made, (2) the number of food and social rewards earned, and (3) the number of pellets either consumed, shared, or left behind. We defined "sharing" as a sequence of behavior consisting of producing food, followed by social release with food remaining, permitting food consumption by the restrained rat. Sharing was possible in all conditions except Condition 5, with no restrained rat present. Food left behind was defined as the difference between the food produced and the total consumed by both rats, and was measured only in Conditions 5 and 7 . It was not possible to measure pellets left behind in Condition 6, as trials continued until all food had been consumed.

\section{Analysis}

Performance was modeled using multi-level generalized linear regression, implemented using the Stan programming language (Carpenter et al. 2017). This Bayesian approach yielded a full estimate of the posterior probability density across all relevant parameters, as well as the behaviors those parameters predict. As such, our analysis does not compare behavior to a null hypothesis; instead, we report the patterns of future behavior that are predicted on the basis of the collected evidence, as well as the uncertainty of those predictions given our statistical models. The analytic scripts used to perform these analyses are included in the electronic supplement, and an annotated summary of the model assumptions is provided in the appendix.

\section{RESULTS}

In order to model the proportion of choices made between food and social reinforcement, a multi-level logistic regression was performed, modeling each subject in each condition with an intercept term. The resulting estimated proportion of responses made by each rat to the food lever in each session of Conditions 1-4 is plotted in Figure 2, as is the estimated mean of the three subjects. When access to food in the home cage was restricted (Conditions 1-3), rats consistently favored the food lever over the social lever, doing so most when each lever press yielded only 1 pellet $(92.4 \%$ mean preference, $\pm 0.4 \%)$, somewhat less when 
Rat 4

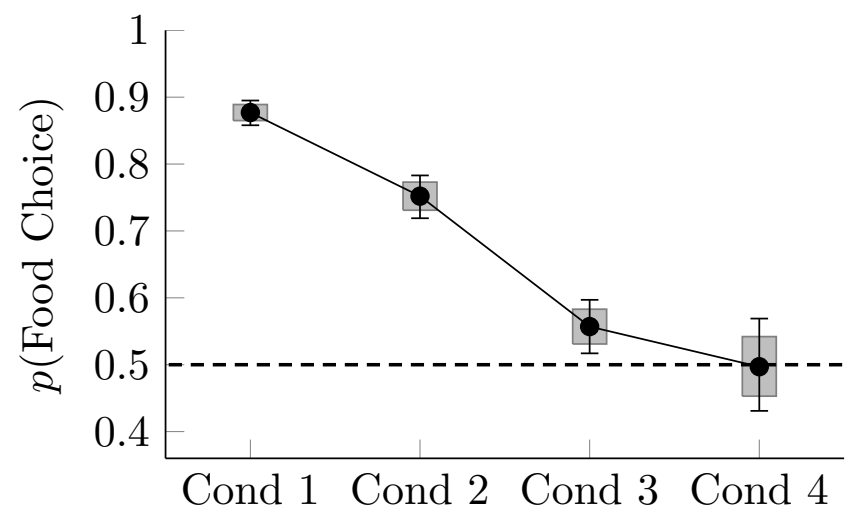

Rat 8

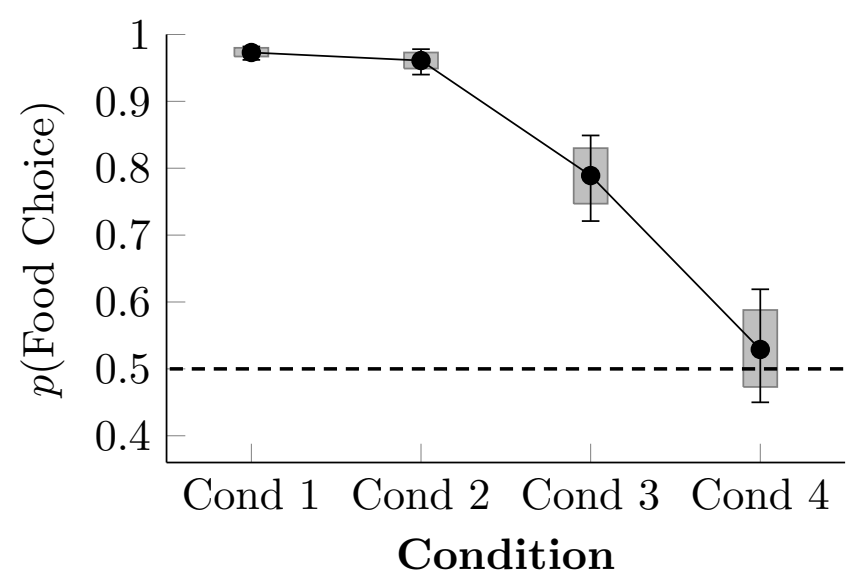

Rat 6

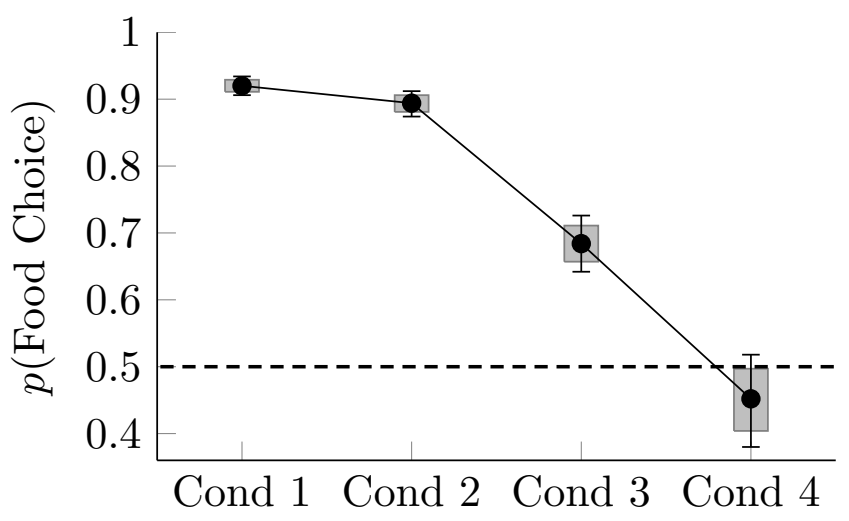

Mean of Subjects

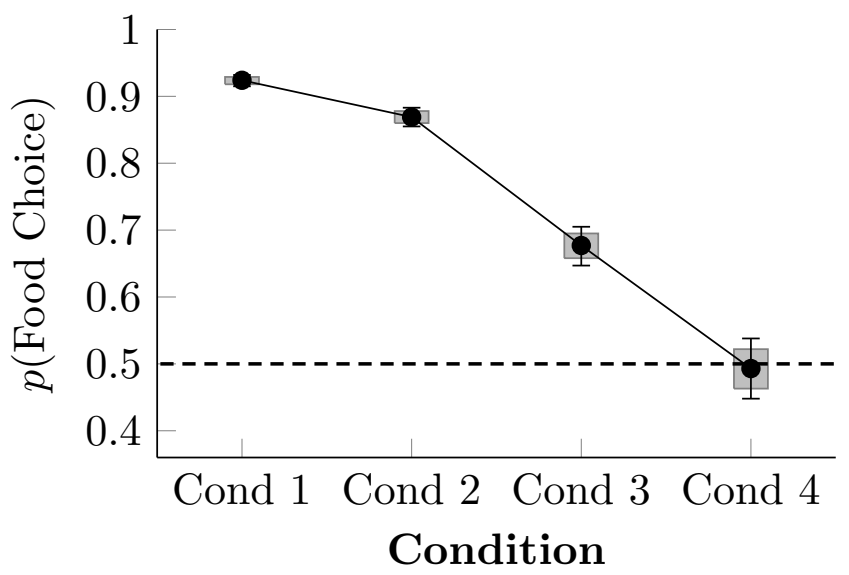

Figure 2. Estimated proportion of food choices made for each of the rats in Conditions 1-4, as well as the mean of the subject estimates, based on a multi-level logistic regression. The proportion of social choices was complementary to the food choices, such that $p$ ("Social Choice") $=1-p$ ("Food Choice"). Points represent posterior means, whereas boxes represent the $80 \%$ credible interval and whiskers represent the $95 \%$ credible interval.

yielding 2 pellets $(86.9 \%$ mean preference, $\pm 0.7 \%)$, and less still when yielding 4 pellets $(67.7 \%$ mean preference, $\pm 1.5 \%$ ). In Condition 4 , with 4 pellets per press and free chow available in the home cage, subjects chose both levers approximately equally over the course of the session (49.3\% mean preference, $\pm 2.3 \%$ ).

While logistic regression provides an estimate of the relative proportion of food and social choices, it does not reveal the absolute rates of responses to each lever. In order to model the estimated number of food and social choices, a multi-level negative binomial regression was implemented (Gelman et al., 2013). This distribution was chosen because we wanted to allow for the possibility that the distributions were overdispersed. Figure 3 plots the estimated number of times each operandum was chosen per session. Food choices decreased both as a function of number of pellets delivered (Conditions 1-3) and also as a function of free access to chow in the home cage (Condition 4). Social choices, however, did not display any consistent pattern across subjects, happening at similar rates across conditions.

Because the rats made frequent food choices and made social choices at consistent rates, there were many opportunities for sharing (that is, for producing food pellets, not consuming them, and then releasing the restrained rat). We estimated the rates of both events with another multi-level negative binomial 

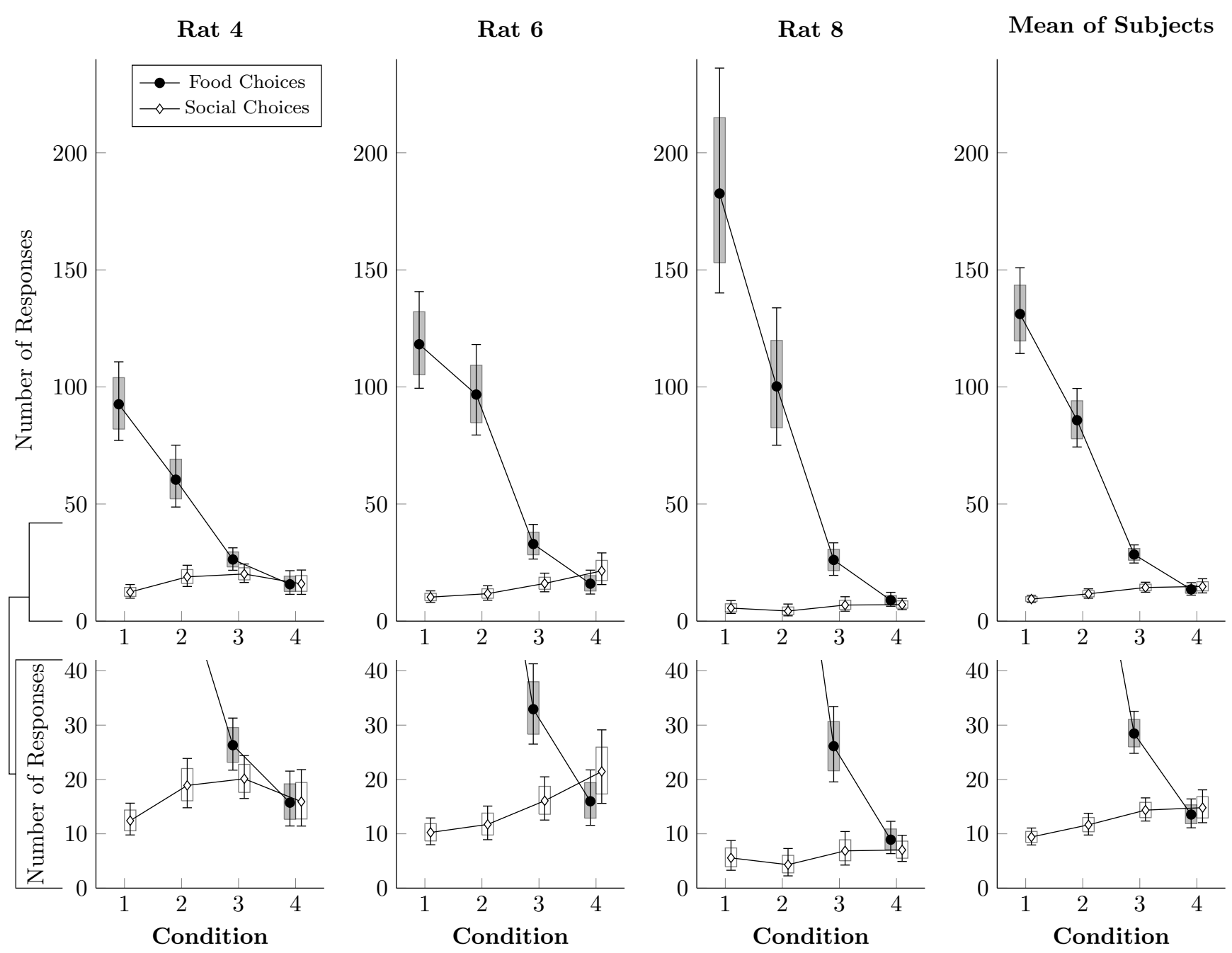

Figure 3. Estimated rates at which food choices (black circles) and social choices (white diamonds) were made for each of the rats in Conditions 1-4, as well as the mean of the subject estimates, based on a multi-level negative binomial regression. Points represent posterior means, whereas boxes represent the $80 \%$ credible interval and whiskers represent the $95 \%$ credible interval.

regression. Figure 4 plots the estimated average frequency with which pellets were either consumed (black circles) or left behind (white diamonds) in a given session. Subjects generally consumed over 100 pellets when access to chow in their home cage was restricted (Conditions 1-3), but consumed around 50 pellets even when they had unrestricted home cage chow (Condition 4). Despite this, the rats effectively never left behind a food pellet in Condition 1 and left behind only one or two pellets in a typical session of Conditions $2-4$. Subjects almost never left behind pellets for the restrained rat to collect, even under circumstances in which pellets could be generated easily and during which the focal rat was not experiencing caloric restriction.

This systematic consumption of food may have been stimulus-driven, insofar as subjects pressing the food lever in Conditions 1-4 had the resulting pellets immediately delivered to the pellet tray mere centimeters away from the lever, and any response to the social lever would require the rat to walk past the tray. In Conditions 5-7, however, pellets had to be collected from the right restraint tube, making the pellet both more laborious to collect and less salient as direct consequences of the lever press. Additionally, Conditions 5-7 featured unrestricted home cage chow, so subjects were less motivated by immediate caloric deficit. 

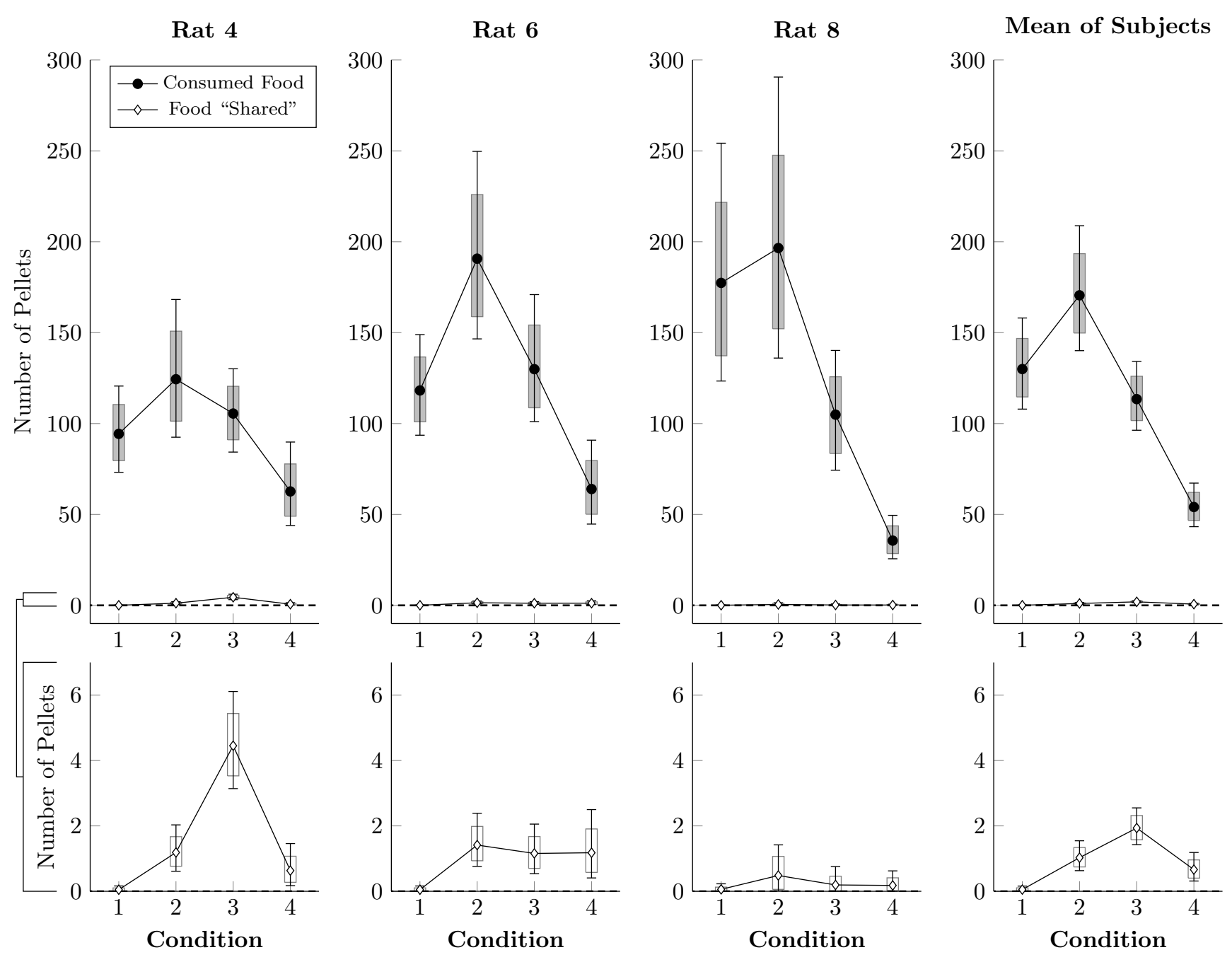

Figure 4. Estimated number of pellets consumed by the focal rat (black circles) and shared with the restrained rat (white diamonds) in Conditions 1-4, as well as the mean of the subject estimates, based on a multi-level negative binomial regression. Points represent posterior means, whereas boxes represent the $80 \%$ credible interval and whiskers represent the $95 \%$ credible interval.

Figure 5 shows preference for food responses relative to social responses in Conditions 6 and 7, as estimated using multi-level logistic regression. (Condition 5 is not present because it lacked a concurrent social schedule.) In general, preference was equivocal, close to 50\% in both Conditions 6 and 7, at rates similar to those seen in Condition 4 (which also featured free feeding in the home cage). On average, the rats were slightly, but probably not meaningfully, more likely to choose food in Condition 7 than in Condition 6 (mean difference of $5.0 \% \pm 3.5 \%$ ).

Figure 6 shows the absolute rates per session of food and social choices, as estimated by multi-level negative binomial regression. Food choices appeared to happen slightly more often in Condition 5, although this is likely due to the absence of a concurrent choice option. Choices in Conditions 6 and 7 resembled those in Condition 4, suggesting that the alternate food delivery paradigm did not substantially alter response rates to either alternative.

Figure 7 shows the average number of pellets consumed, shared, or left behind (i.e. consumed by neither rat) in Conditions 5-7, as estimated by multi-level negative binomial regression. As in the earlier conditions, the focal rat tended to consume the vast majority of pellets, but also left quite a few pellets 
Rat 4

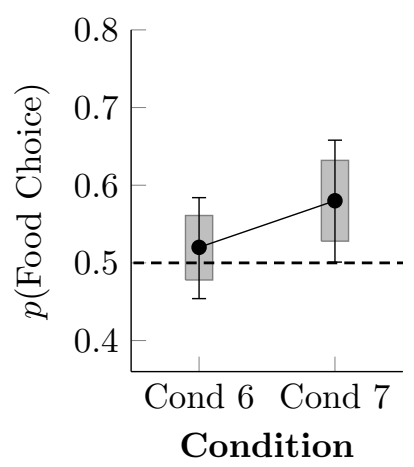

Rat 6

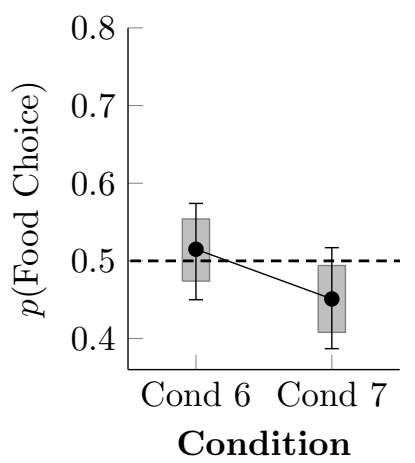

Rat 8

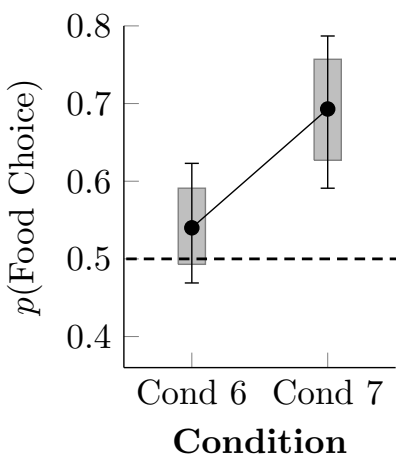

Mean of Subjects

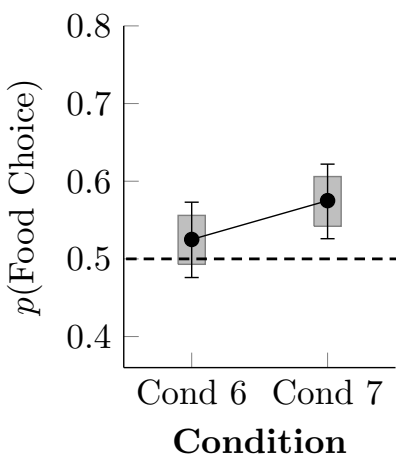

Figure 5. Estimated proportion of food choices made for each of the rats in Conditions 6 and 7, as well as the mean of the subject estimates, based on a multi-level logistic regression. The proportion of social choices was complementary to the food choices, such that $p$ ("Social Choice") $=1-p$ ("Food Choice"). Points represent posterior means, whereas boxes represent the $80 \%$ credible interval and whiskers represent the $95 \%$ credible interval.

behind due to the time limit on the food collection period in Conditions 5 and 7 . In general, more pellets were left behind in Condition 5 (6.0 mean pellets, \pm 1.0 ), than in Condition 7 (3.3 mean pellets, \pm 0.5 ). Sharing, by contrast, happened at low rates in Conditions 6 and 7, comparable to the earlier conditions (1-4). Even given unlimited time to collect pellets, few were shared in Condition 6 (1.5 mean pellets, $\pm 0.3)$, and with a 30-s time limit on their collection, even fewer were shared in Condition 7 (0.6 mean pellets, \pm 0.2$)$.

\section{DISCUSSION}

The present experiment was designed to replicate and extend some key conditions described by BenAmi Bartal et al. (2011), in which rats chose between social release and food. The present research focused on two main findings from that study and their related conclusions: (1) rats chose food and social release with similar latencies, and therefore, food and social release are equally valued; and (2) rats willingly share food with their social partner, even if it comes at a cost to the individual. Taken together, these findings provide key support for the authors' claims of altruistic food sharing. Because occurrences of such unreciprocated food sharing are rare in the published literature (Clutton-Brock, 2009; Taborsky et al., 2016), they warrant further scrutiny.

With respect to the first claim of equal reward value of social release and food, we found that relative value of food and social release varied systematically across conditions. More specifically, when food motivation was low (i.e., the focal rat had unrestricted homecage access to chow in their home cage) and food quantity was high (4-5 pellets per trial), food and social release were chosen about equally often (Conditions 4, 6, and 7), consistent with the Ben-Ami Bartal et al. (2011) findings. When food motivation was high (restricted access to food outside the session), however, rats clearly preferred food over social release (Conditions 1-3). This finding is consistent with the Hiura et al. (2018) findings, showing strong and reliable preference for food over social release when food is restricted outside the session (see also Blystad et al., 2019). Taken as a whole, the presents results show that relative preference between social and food is not invariant, but rather, is subject to reward and motivational variables (food quantity and overall food access). The relative value of social release and food are always subject to these (and other) variables, and it would therefore be premature to draw broad conclusions about their relative value from sampling only a limited range of conditions.

The changes in preference across manipulation of food quantity in the first three conditions were 

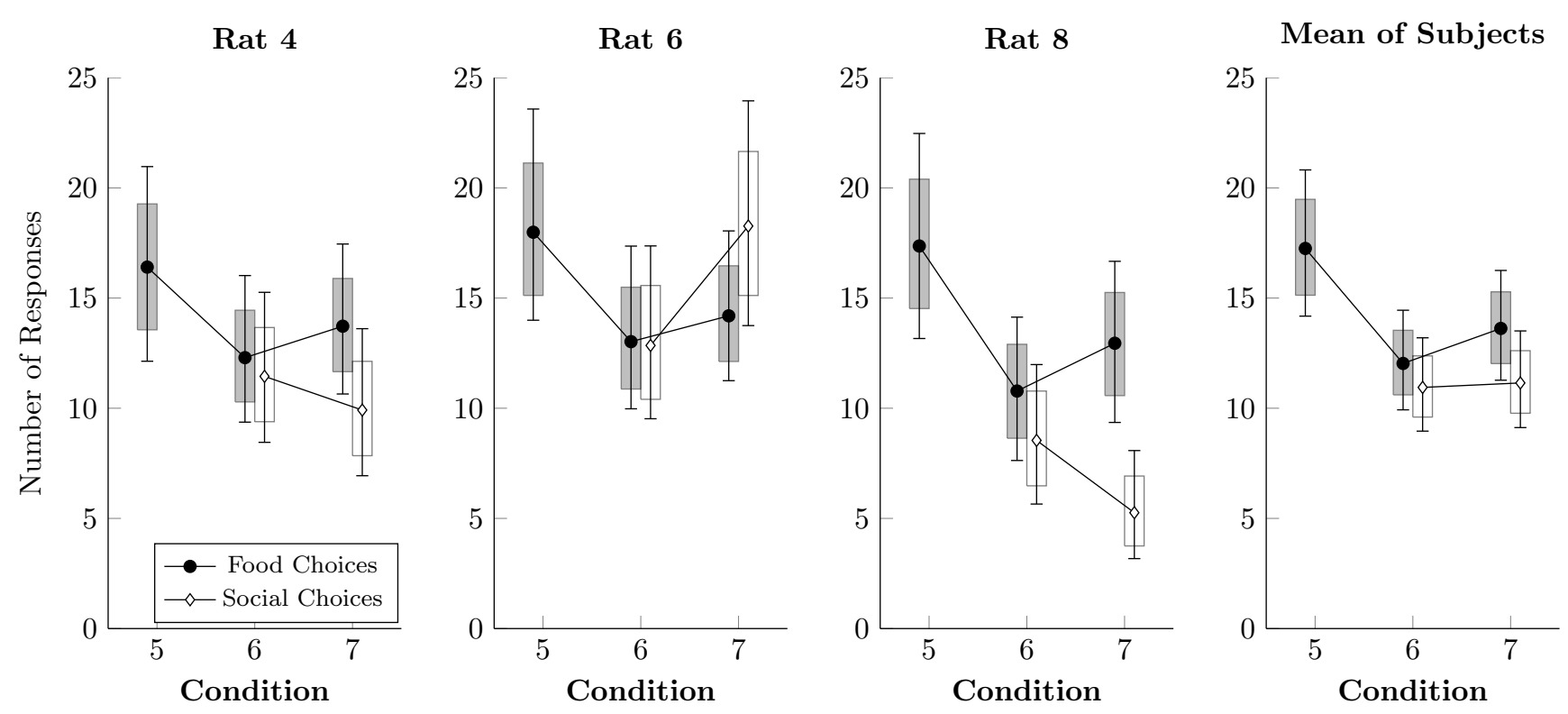

Figure 6. Estimated rates at which food choices (black circles) and social choices (white diamonds) were made for each of the rats in Conditions 5-7, as well as the mean of the subject estimates, based on a multi-level negative binomial regression. Points represent posterior means, whereas boxes represent the $80 \%$ credible interval and whiskers represent the $95 \%$ credible interval.

driven mainly by changes in the number of food choices per session. This is partly due to economic factors (i.e., decreasing unit price of food) and partly due to satiation. Given the low price (1 response) and the dozens of choice opportunities each session, rats produced and consumed large numbers of sucrose pellets each session when chow in their home cage was restricted (37-284 pellets across rats, mean $=$ 131). By contrast, when home cage chow was unlimited and food motivation was low, subjects consumed substantially fewer pellets $(16-107$ pellets across rats, mean =66). And when coupled with unlimited food access outside the session in Condition 4, the procedures combined to produce conditions of low food need. Indeed, our rats had such an abundance of food, there was often food left at the end of the food collection periods of Conditions 5-7 (up to 26\% of all pellets in Condition 5), even if there was no restrained rat with which to share them. That rats did not consume rewards as highly valued as sucrose pellets suggests a high degree of satiation.

Despite such low levels of food need, there was very little evidence of food sharing — the second and more controversial claim set forth by Ben-Ami Bartal et al. (2011). Behavior that met our operational definition of sharing (i.e., producing food and then releasing the rat while food remained available) was infrequent across all conditions in the experiment, with zero shared pellets being the most common outcome across sessions and the mean being about 1 pellet per session. It did not matter whether food access outside the session was restricted (Conditions 1-3) or not (Conditions 4-7); nor did it matter how many pellets were produced per response (Condition 1-3): rats rarely shared with the other rat any of the abundant supply of food pellets they produced each session. Even in the final two conditions, with procedures that most closely matched the original study (i.e., symmetrically arranged social and food locations, 5 sucrose pellets, and unrestricted access to food and social contact outside the session), sharing was seldom observed. Thus, on the whole, we found no evidence to support the 2011 claim by Ben-Ami Bartal et al. that a rat willingly shares food with another rat.

There is no simple way to reconcile the food sharing reported by Ben-Ami Bartal et al. (2011) with the near complete absence of sharing in the present study. Low levels of food sharing cannot be explained in terms of reduced opportunities for sharing, as the number of social releases (hence, sharing opportunities) 
Rat 4

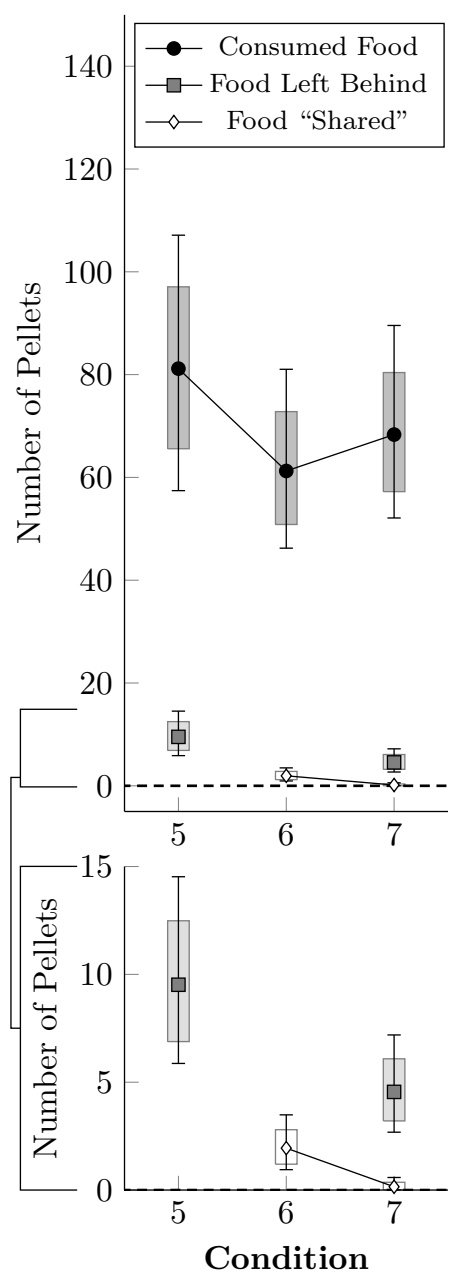

Rat 6
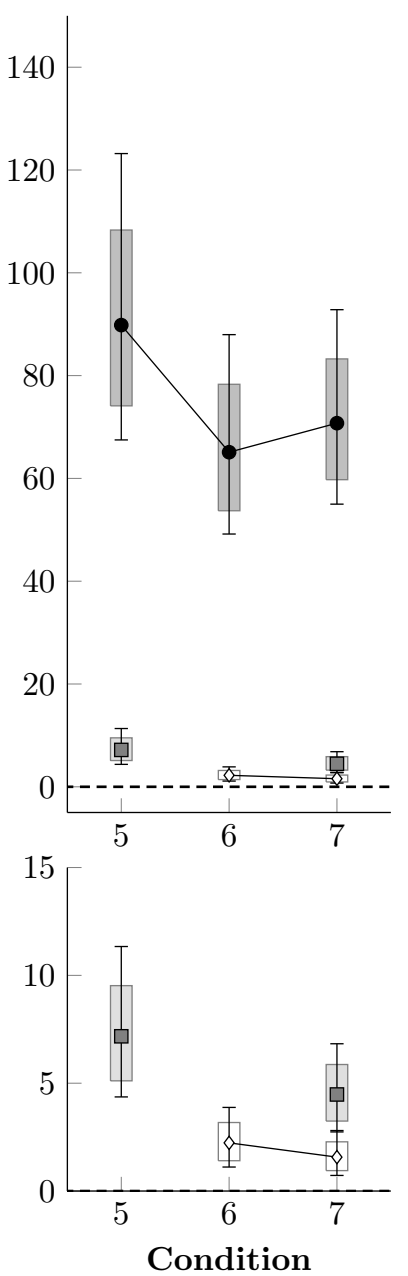

Rat 8
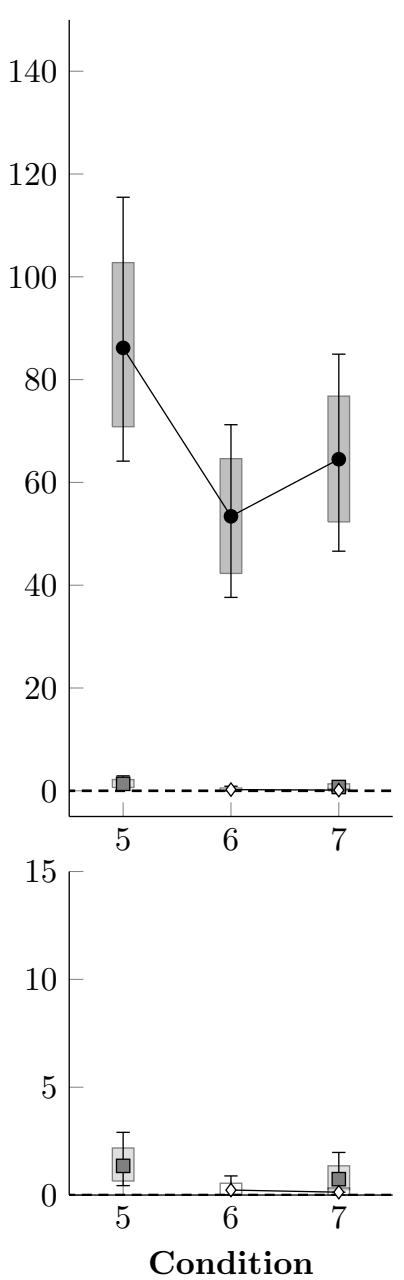

Mean of Subjects
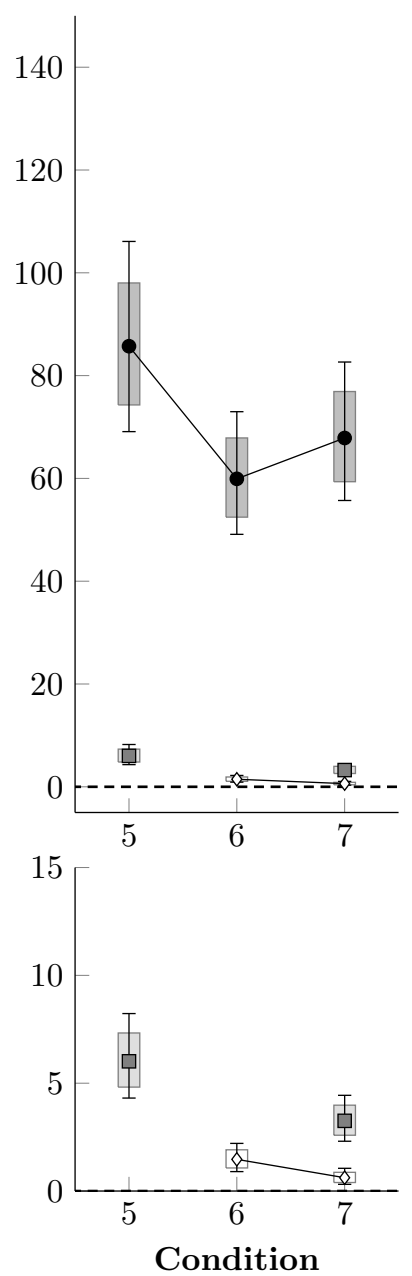

Figure 7. Estimated number of pellets consumed by the focal rat (black circles), shared with the restrained rat (white diamonds), or left unconsumed by either rat (gray squares) in Conditions 5-7, as well as the mean of the subject estimates, based on a multi-level negative binomial regression. Points represent posterior means, whereas boxes represent the $80 \%$ credible interval and whiskers represent the $95 \%$ credible interval.

remained fairly constant across conditions for individual rats (see Figures 3 and 6). This was accomplished by providing repeated exposure to a consistent duration of social contact (30 s) across the experiment. With long sessions and repeated trials, rats had ample opportunities to share the food they had produced; they simply did not do so.

The discrepant results also cannot be explained in terms of differing definitions of sharing between experiments. Ben-Ami Bartal et al. (2011) used a less stringent indirect measure of sharing (difference between food consumed with and without a rat available to release) than our behavioral definition of sharing (produce food, then social release with food remaining). This alone cannot be responsible for the different results, however, for even if we adopt the less stringent criterion, our rats showed no differences in food consumption with or without a rat available to release (Figure 7). This is important, as evidence of sharing-related costs are crucial to an altruistic food sharing explanation. Thus, by neither definition did our rats engage in sharing.

We recognize that the present study relies on a small sample size of three rats. Even so, however, the evidence against food sharing is strong. Across all of the conditions in which sharing was possible, 
our rats earned an average of 2,171 rewards each (1,662-2,772, across rats) of which they shared an average of 47 ( $0-85$, across rats), or $1 \%$ of the total rewards earned. Because each earned reward provided a sharing opportunity, our rats had vastly greater food sharing opportunities than rats in the Ben-Ami Bartal et al. (2011) experiment. Precise estimates of food sharing opportunities in that experiment are difficult, both because opportunity per reward cannot be derived from the data presented in the paper (\% trials with sharing), and because it took the rats several sessions to learn to open the door for either option (before which rewards were not actually available to share). Nonetheless, the theoretical maximum would be 60 food sharing opportunities (five rewards per trial for 12 trials) per rat, roughly $1 \%$ of the number food-sharing opportunities in the present study. Thus, while the small number of rats in the present study limits our ability to generalize to the population of all rats, we have considerable confidence in the results with these particular rats: all were strongly disinclined to share food with their partners across all conditions and thousands of sharing opportunities. Perhaps only some rats engage in altruistic food sharing, differing from their non-sharing conspecifics for some reasons yet to be discovered, and that our sample happened to include only selfish rats. This seems unlikely, but it will nevertheless be important to replicate with larger samples of rats in future research.

Another difference between the studies is the food itself. Ben-Ami Bartal and colleagues used a single presentation of 5 chocolate chips, which amounts to approximately 12 calories of food, contained in about $1.62 \mathrm{~g}$. By comparison, each of our sucrose pellets constitutes approximately 0.17 calories, each with a mass of $0.045 \mathrm{~g}$. When subjects had unlimited home cage access to chow, they therefore tended to consume about 11.2 calories of food. Furthermore, rats in Condition 5 left about 6 pellets (worth about 1 calorie) behind, despite having no external motivation to do so. This points to subjects with free access to chow leaving high-quality food unconsumed due to satiation, usually doing so shy of 12 calories. If rats with low food motivation are inclined to leave food unconsumed relatively frequently in the absence of conspecifics, it is difficult to argue that losing such food due to sharing can be understood as a "cost." Ben-Ami Bartal and colleagues give no rationale for their choice of 5 chocolate chips, but based on the patterns of non-social food intake observed in the present study, it seems likely that, had they used 3 chocolate chips, that would have observed almost no sharing, whereas if they had used 7 chocolate chips, they would have observed relatively frequent sharing.

There are other differences between the procedures, and the only way to know for certain which factors are responsible for the discrepant results would be to begin with a direct replication, an exact reproduction of the original procedures, and thereafter change one variable at a time. We chose instead to conduct a systematic replication (Sidman, 1960), in which some, but not all, of the original procedures are reproduced. Systematic replications are useful in assessing the generality of a finding, and this fit with our broader objectives of providing a more thorough characterization of preference and sharing. We sought not only to replicate but to extend, to assess the generality of the findings by exploring behavior across a range of conditions, including but not limited to, those of the original study. In particular, the lack of adequate control conditions leaves the original study open to multiple interpretations. Sampling independent variables under varying conditions puts replication efforts into a broader context, changing the focus from binary questions with yes-no answers (e.g., Do rats value social release over food? Do rats share food with another rat?) toward conditional questions (e.g., Under what conditions is social release favored over food, and vice versa? Under what conditions does sharing occur?). Viewed in this way, Ben-Ami Bartal and her collaborators are not so much incorrect as they are interpreting incomplete evidence; their results are part of more general relationships between preference and sharing and the variables of which they are a function.

Exploring such functional relationships across a parametric range can also shed light on theoretical disputes. For example, when examined at only a single point on a function, social release can be interpreted 
either in terms of social reward (response-contingent access to social interaction) or in terms of empathy (acting out of concern for the other rat): both accounts make the same prediction that door opening will occur. The accounts begin to differ, however, as behavior is examined as other experimental parameters change. For example, in procedures similar to those used here, Vanderhooft et al. (2019) first trained social release in rats, then increased the price of social release (number of responses to produce it) systematically increased across sessions, generating demand functions. Overall, the functions (27 in all) were well described by the Hursh and Silberberg (2008) essential value model, a model that has proven useful in quantifying the value of numerous other rewards, including food, water, and drugs Hursh and Roma (2016). In other words, these functions were predictable, with a high degree of quantitative precision, on the basis of social reward functions. It is less clear, however, what, if anything, an empathy account would have to say about these data: it makes no obvious predictions about how empathy is affected by price - or other variables known to affect reward value (e.g., magnitude, delay, or probability), about which social reward makes clear and testable predictions. And if predictions could be derived from an empathy account (e.g., by assuming that empathy mirrors social reward functions), they would be indistinguishable from the more parsimonious social reward account, and would therefore add little to the explanation. This is not to deny the importance of empathy as a topic worthy of scientific study; it is, rather, to demand more stringent tests of it, especially in domains in which simpler explanations already exist.

\section{APPENDIX: MODEL SPECIFICATIONS}

Each of the following annotated model summaries describes the statistical assumptions made by our models, using the notation described by Gelman et al. (2013).

\section{Model for Proportion of Food Choices (Figures 2 \& 5)}

$F_{i} \sim \operatorname{Binomial}\left(N_{i}, p_{s, c}\right)$

The number of food choices $F_{i}$ made out of $N_{i}$ opportunities is governed by a binomial distribution with a probability $p_{s, c}$, which is a separate value for each subject in each condition.

$p_{s, c} \sim \operatorname{Logistic}\left(\beta_{s, c}\right)$

The parameters describing subjects' performance are encoded as unbounded scalar parameters that are converted to probabilities by the logistic function.

$\boldsymbol{\beta} \sim \operatorname{MVNormal}(\boldsymbol{\mu}, \boldsymbol{\Sigma})$

$\boldsymbol{\beta}$ is a Subjects-by-Conditions matrix whose values are drawn from a multivariate normal distribution. A subject's mean across all conditions is given by the corresponding element in the vector of means $\boldsymbol{\mu}$.

$\mu_{s} \sim \operatorname{Normal}(0,1.5)$

The prior for each subject's mean $\mu_{s}$ is normally distributed.

$\Sigma \sim \operatorname{diag}(\boldsymbol{\tau}) \cdot \operatorname{LkjCorr}(2) \cdot \operatorname{diag}(\boldsymbol{\tau})$

The covariance matrix $\Sigma$ is obtained by construction using a vector of magnitudes $\tau$ and the LKJ Correlation distribution (Lewandowski et al. 2009).

$\tau_{s} \sim$ Exponential $(1.5)$

The prior for each subject's covariance magnitude $\tau_{s}$ is exponentially distributed. 


\section{Model for Proportion of Food Choices (Figures 3 \& 6)}

$R_{i} \sim \operatorname{NegBinomial}\left(\mu_{s, c, t}, \phi\right)$

The total number of responses $R_{i}$ is governed by a negative binomial distribution using the alternate parameterization whose mean is $\mu$ and whose variance is $\mu+\frac{\mu^{2}}{\phi}$. Each subject has two parameters in each condition: One governing food responses and one governing social responses (if available).

$\mu_{s, c, t} \sim \log \left(\beta_{s, c, t}\right)$

The parameters describing subjects' performance are encoded as unbounded scalar parameters that are made strictly positive by a log transformation.

$\boldsymbol{\beta} \sim \operatorname{MVNormal}(\boldsymbol{\gamma}, \boldsymbol{\Sigma})$

$\boldsymbol{\beta}$ is a Subjects-by-(Conditions $\times 2)$ matrix whose values are drawn from a multivariate normal distribution, such that each condition yields two parameters per subject (one for food responses and one for social responses). A subject's mean across all conditions is given by the corresponding element in the vector of means $\gamma$.

$\gamma_{s, t} \sim \operatorname{Normal}(1,1.5)$

The prior for each subject's mean $\gamma_{s, t}$ is normally distributed.

$\Sigma \sim \operatorname{diag}(\boldsymbol{\tau}) \cdot \operatorname{LkjCorr}(2) \cdot \operatorname{diag}(\boldsymbol{\tau})$

The covariance matrix $\Sigma$ is obtained by construction using a vector of magnitudes $\tau$ and the LKJ Correlation distribution (Lewandowski et al. 2009).

$\tau_{s, t} \sim$ Exponential (1.5)

The prior for each subject's covariance magnitude $\tau_{s, t}$ is exponentially distributed.

$\phi \sim \operatorname{Exponential~(1)~}$

The negative binomial overdispersion term has an exponential prior and is shared across subjects.

\section{Model for Proportion of Food Choices (Figures 4 \& 7)}

The model for counting the total pellets either consumed, shared, or left behind followed identical logic to the negative binomial regression described above, with two exceptions. First, it provided three parameters per subject per condition, rather than two, in order to account for the three different observed counts. Second, the prior for $\gamma_{s}$ was set to Normal $(0,1.5)$ in anticipation of the lower counters for all three cases.

\section{Acknowledgments}

We are indebted to Greg Wilkinson for his expert technical assistance, to Melanie Meredith for help conducting the sessions, and to Francesca degli Espinosa for comments on an earlier version of the manuscript. Portions of these data were presented at the 2019 meeting of the Association for Behavior Analysis International. 


\section{Funding}

The research was funded by Reed College Summer Scholarship Funds.

\section{Author Contributions}

The study was designed by HW, CK, \& TDH. Data were collected by HW \& CK. Data were analyzed by HW, CK, \& GJ. The paper was written by HW, CK, GJ, \& TDH.

\section{REFERENCES}

Ben-Ami Bartal, I., Decety, J., and Mason, P. (2011). Empathy and pro-social behavior in rats. Science, 334:1427-1430.

Ben-Ami Bartal, I., Rodgers, D. A., Bernadez Sarria, M. S., Decety, J., and Mason, P. (2014). Pro-social behavior in rats is modulated by social experience. ELife, 3:e01385.

Blystad, M. H., Andersen, D., and Johansen, E. B. (2019). Female rats release a trapped cagemate following shaping of the door opening response: Opening latency when the restrainer was baited with food, was empty, or contained a cagemate. PLOS ONE, 14:e0223039.

Clutton-Brock, T. (2009). Cooperation between non-kin in animal societies. Nature, 462:51-57.

Cronin, K. A. (2012). Prosocial behaviour in animals: The influence of social relationships, communication and rewards. Animal Behaviour, 84:1085-1093.

Gelman, A., Crlin, J. B., Stern, H. S., Dunson, D. B., Vehtari, A., and Rubin, D. B. (2013). Bayesian Data Analysis, 3rd Edition. CRC Press, Boca Raton, FL, USA.

Hachiga, Y., Schwartz, L. P., Silberberg, A., Kerns, D. N., Gomez, M., and Slotnick, B. (2018). Does a rat free a trapped rat due to empathy or for sociality? empathy versus sociality. Journal of the Experimental Analysis of Behavior, 110:267-274.

Hiura, L. C., Tan, L., and Hackenberg, T. D. (2018). To free, or not to free: Social reinforcement effects in the social release paradigm with rats. Behavioural Processes, 152:37-46.

Hursh, S. R. and Roma, P. G. (2016). Behavioral economics and the analysis of consumption and choice. Managerial and Decision Economics, 37:224-238.

Hursh, S. R. and Silberberg, A. (2008). Economic demand and essential value. Psychological Review, 115:186-198.

Sato, N., Tan, L., Tate, K., and Okada, M. (2015). Rats demonstrate helping behavior toward a soaked conspecific. Animal Cognition, 18:1039-1047.

Schwartz, L. P., Silberberg, A., Casey, A. H., Kerns, D. N., and Slotnick, B. (2017). Does a rat release a soaked conspecific due to empathy? Animal Cognition, 20:299-308.

Sidman, M. (1960). Tactics of Scientific Research: Evaluating Experimental Data in Psychology. Basic Books, New York, NY, USA.

Silberberg, A., Allouch, C., Sandfort, S., Kearns, D., Karpel, H., and Slotnick, B. (2014). Desire for social contact, not empathy, may explain "rescue" behavior in rats. Animal Cognition, 17:609-618.

Sosnowski, M. J. and Brosnan, S. F. (2019). Pro-social behavior. In Vonk, J. and Shackelford, T., editors, Encyclopedia of Animal Cognition and Behavior. Springer International Publishing.

Taborsky, M., Frommen, J. G., and Riehl, C. (2016). Correlated pay-offs are key to cooperation. Philosophical Transactions of the Royal Society B: Biological Sciences, 371:20150084.

Vanderhooft, L., Tan, L., and Hackenberg, T. (2019). Demand for social contact in rats: Toward a quantitative analysis of social reinforcement value. Mexican Journal of Behavior Analysis, 45.

West, S. A., Griffin, A. S., and Gardner, A. (2007). Social semantics: Altruism, cooperation, mutualism, strong reciprocity and group selection. Journal of Evolutionary Biology, 20:415-432. 